TITLE:

\title{
Preconditioning based on Calderon's formulae for periodic fast multipole methods for Helmholtz' equation
}

$\operatorname{AUTHOR}(\mathrm{S}):$

Niino, Kazuki; Nishimura, Naoshi

\section{CITATION:}

Niino, Kazuki ... [et al]. Preconditioning based on Calderon's formulae for periodic fast multipole methods for Helmholtz' equation. Journal of Computational Physics 2012,231(1): 66-81

ISSUE DATE:

2012-01

URL:

http://hdl.handle.net/2433/150932

\section{RIGHT:}

(C) 2011 Elsevier Inc.; This is not the published version. Please cite only the published version.; この論文は出版社版でありません。引用の際に は出版社版をご確認ご利用ください。 


\title{
Preconditioning based on Calderon's formulae for periodic fast multipole methods for Helmholtz' equation
}

\author{
Kazuki NIINO ${ }^{\text {a }}$, Naoshi NISHIMURA ${ }^{\text {b }}$ \\ ${ }^{a}$ Graduate School of Informatics, Kyoto Univ.niino@acs.i.kyoto-u.ac.jp \\ ${ }^{b}$ Graduate School of Informatics, Kyoto Univ.nchml@i.kyoto-u.ac.jp
}

\begin{abstract}
Solution of periodic boundary value problems is of interest in various branches of science and engineering such as optics, electromagnetics and mechanics. In our previous studies we have developed a periodic Fast Multipole Method (FMM) as a fast solver of wave problems in periodic domains. It has been found, however, that the convergence of the iterative solvers for linear equations slows down when the solutions show anomalies related to the periodicity of the problems. In this paper, we propose preconditioning schemes based on Calderon's formulae to accelerate convergence of iterative solvers in the periodic FMM for Helmholtz' equations. The proposed preconditioners can be implemented more easily than conventional ones. We present several numerical examples to test the performance of the proposed preconditioners. We show that the effectiveness of these preconditioners is definite even near anomalies.
\end{abstract}

Keywords: BEM, FMM, Preconditioning, Calderon's formulae, Wood's anomaly

\section{Introduction}

In science and engineering, one finds various interesting applications of periodic structures which utilise peculiar behaviours of waves in periodic domains. In optics, for example, one can mention photonic crystals [1] which show various interesting features such as band gap structures, and metamaterials [2] which may be designed to have an apparently negative refractive index, etc. It is important to develop fast solvers of periodic wave scattering problems to analyse such structures efficiently. Although the finite difference time domain (FDTD) method is a well-accepted solver for such problems, the 
boundary element method (BEM), enhanced with the fast multipole method (FMM), is also considered to be an effective alternative[3, 4].

Indeed, we can expect that the number of unknowns $N$ is small in BEM because we need to discretise only the boundaries of domains. Although the inefficiency of BEM due to its $O\left(N^{2}\right)$ complexity has been a long standing problem, we can now reduce the $O\left(N^{2}\right)$ computational time of the original BEM to $O\left(N(\log N)^{\alpha}\right)(\alpha \geq 0)$ with the help of FMM. Furthermore, since the algorithm of FMM utilises the division of the domain of problems into cubic "boxes", it can efficiently deal with periodic problems as we regard the unit cell (unit of the periodicity) as the level 0 box of FMM. The combination of BEM and FMM is therefore considered to be suitable as a solver of scattering problems for periodic structures.

The computational time of FMM now depends mainly on the iteration number of the linear equation solver because the linear equation is usually solved with iterative solvers. This is why the developments of good preconditioners is very important in the study of FMMs. This is all the more so in periodic scattering problems where the solutions are known to show anomalous behaviour occasionally[5]. Indeed, the phenomena known as Wood's anomalies are characterised by sudden changes of solutions in response to slight changes of the wave lengths or the incident angles of the incident waves. These anomalies are related to the existence of guided modes, leaky modes, cutoff frequencies, etc., which are typically seen in the solutions of periodic wave problems. It has been shown that the convergence of iterative solvers in periodic FMMs degrades near these anomalies (Otani and Nishimura [6]).

In passing, we would like to point out that there exists confusion related to the definition of 'Wood's anomalies' in literature. Indeed, in engineering, Wood's anomalies refer to physical phenomena stated above. In mathematics, however, Wood's anomalies stand for the cutoff frequencies where the periodic Green's function diverges. The same phenomena are usually called 'Rayleigh's anomalies' in engineering. We here follow the engineering practice to use the expression 'Wood's anomaly' in a physical sense, and refer to what mathematicians call 'Wood's anomaly' as 'Rayleigh's anomaly'.

In the Krylov subspace methods such as GMRES and BiCG, we can decrease the iteration number by multiplying the coefficient matrix by matrices called preconditioners[7]. Among various preconditioners which have been proposed in the past, the one based on Calderon's formulae is considered promising. This type of preconditioner was proposed by Steinbach and Wendland for Laplace's equation[8]. Christiansen and Nédélec later applied 
this preconditioning to Helmholtz' equation[9]. Antoine and Boubendir found that this preconditioning is particularly effective for the problems in multiple domains and numerically verified its effectiveness for non-periodic problems for Helmholtz' equation in 2D[10]. Also noteworthy are the recent developments in the Calderon preconditioners for Maxwell's equations in which the use of Buffa-Christiansen basis [11] together with the conventional RWG (or Raviart-Thomas) basis is considered (see $[12,13]$ for example).

In our previous study, we examined the Calderon preconditioning in periodic transmission problems for Helmholtz' equation in 2D and found that the good performance of the Calderon preconditioning can be exploited just by ordering equations and unknowns appropriately in the BEM equations obtained with collocation[14] (See section 3.2 for a brief summary of observations made in [14]). However, we have not so far addressed important issues such as the effect of anomalies, Galerkin discretisation or 3 spatial dimensionality.

In this paper, we present a preconditioning based on Calderon's formulae for periodic transmission problems for Helmholtz' equation in 3D. We consider the Galerkin discretisation which is often used with hypersingular integral equations. Our findings can be summarised as follows; The Gram matrix associated with the basis functions is effective as a preconditioner for the Galerkin matrices, although we cannot fully precondition them just by ordering the unknowns and equations appropriately as we could in the collocation case. This preconditioner is sparse, symmetric, and is easy to invert iteratively. We also verify numerically that the proposed preconditioning based on Calderon's formulae is effective even near anomalies.

This paper is organised as follows. In section 2, we formulate periodic transmission problems governed by Helmholtz' equation in 3D and the associated BEM solved with Galerkin's method. We then investigate preconditioning schemes based on Calderon's formulae in section 3. After showing some numerical examples in section 4 , we summarise this paper and present future plans in section 5 .

\section{Periodic boundary value problems for Helmholtz' equation in $3 \mathrm{D}$}

In this section, we formulate periodic boundary value problems and a boundary element method to solve them. 


\subsection{Governing equation}

We define the domain $D \subset \mathbb{R}^{3}$ by

$$
D=(-\infty, \infty) \otimes[-L / 2, L / 2] \otimes[-L / 2, L / 2] .
$$

The domain $D$ is divided into $N_{d}$ disjoint subdomains such that $D=\overline{D_{1} \cup D_{2} \cup \cdots \cup D_{N_{d}}}$ holds (Fig. 1). The subdomain $D_{1}$ is an infinite domain while others are finite domains.

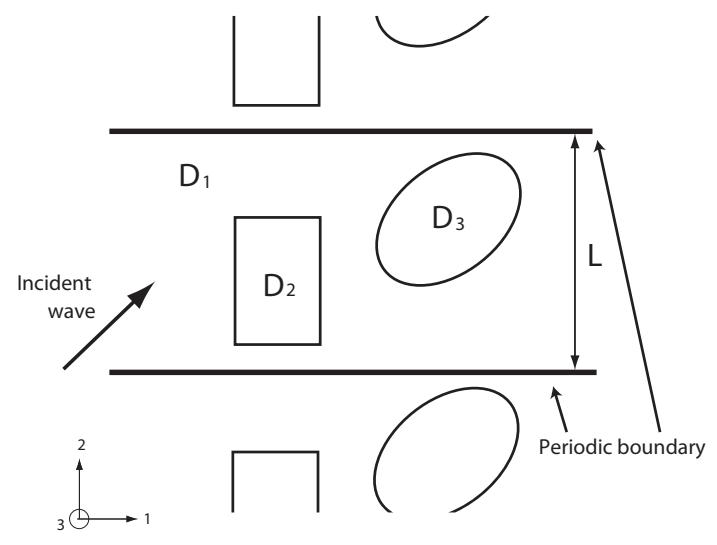

Figure 1: Periodic boundary value problems.

Now, we consider the problem of finding a function $u$ satisfying the Helmholtz equation:

$$
\Delta u+k_{i}^{2} u=0
$$

in every subdomain $D_{i}$. The function $u$ also satisfies boundary conditions:

$$
\begin{aligned}
u_{i} & =u_{j} \\
\frac{1}{\varepsilon_{i}} \frac{\partial u_{i}}{\partial n} & =\frac{1}{\varepsilon_{j}} \frac{\partial u_{j}}{\partial n}
\end{aligned}
$$

on $\partial D_{i} \cap \partial D_{j}$, periodic boundary conditions:

$$
\begin{gathered}
u\left(x_{1}, L / 2, x_{3}\right)=\mathrm{e}^{\mathrm{i} \beta_{2}} u\left(x_{1},-L / 2, x_{3}\right) \\
u\left(x_{1}, x_{2}, L / 2\right)=\mathrm{e}^{\mathrm{i} \beta_{3}} u\left(x_{1}, x_{2},-L / 2\right) \\
\frac{\partial u}{\partial x_{2}}\left(x_{1}, \frac{L}{2}, x_{3}\right)=\mathrm{e}^{\mathrm{i} \beta_{2}} \frac{\partial u}{\partial x_{2}}\left(x_{1},-\frac{L}{2}, x_{3}\right) \\
\frac{\partial u}{\partial x_{3}}\left(x_{1}, x_{2}, \frac{L}{2}\right)=\mathrm{e}^{\mathrm{i} \beta_{3}} \frac{\partial u}{\partial x_{3}}\left(x_{1}, x_{2},-\frac{L}{2}\right)
\end{gathered}
$$


and the scattered field $u^{s}=u-u^{\mathrm{I}}$ satisfies the radiation condition in $D_{1}$, where $u_{i}$ is the limit value of $u$ from domain $D_{i}$ to $\partial D_{i}, k_{i}$ is the wave number in $D_{i}, u^{\mathrm{I}}$ is the incident wave and $\varepsilon_{i}$ is a constant defined in each subdomain $D_{i}$. Also, $\boldsymbol{n}$ is the normal vector defined on $\partial D_{i}$ and the direction of $\boldsymbol{n}$ is fixed. We assume $u^{\mathrm{I}}$ to be a plane wave given as follows unless stated otherwise:

$$
u^{\mathrm{I}}(\boldsymbol{x})=C_{\text {inc }} \mathrm{e}^{\mathrm{i} k_{1} \boldsymbol{v} \cdot \boldsymbol{x}}
$$

where $C_{\text {inc }} \in \mathbb{C}$ is a constant and $\boldsymbol{v} \in \mathbb{R}^{3}$ is a unit vector which represents the incident direction. The numbers $\beta_{i} \in \mathbb{R}(i=2,3)$ which appear in (3),(4), (5) and (6) are the phase differences of the incident wave between $x_{i}=-L / 2$ and $x_{i}=L / 2$, expressed by $\beta_{i}=k_{1} v_{i} L(i=2,3) \bmod 2 \pi$ where $v_{i}$ is the $i$ th component of $\boldsymbol{v}$. Note that $k_{1}$ is not the first component of the wave vector but the wave number in the domain $D_{1}$.

\subsection{Boundary integral equation}

The solution to the above problem is well-known to have the following expression for $\boldsymbol{x} \in D_{j}$ :

$$
u_{j}(\boldsymbol{x})=\delta_{j 1} u^{\mathrm{I}}(\boldsymbol{x})-\left\{\varepsilon_{j} \mathcal{S}^{j}\left(\operatorname{sgn}_{j} q_{j}\right)-\mathcal{D}^{j} u_{j}\right\}(\boldsymbol{x})
$$

where $\mathcal{S}^{j}$ and $\mathcal{D}^{j}$ are defined by

$$
\begin{aligned}
& \left(\mathcal{S}^{j} v\right)(\boldsymbol{x})=\int_{\partial D_{j}} G_{j}^{\mathrm{p}}(\boldsymbol{x}-\boldsymbol{y}) v(\boldsymbol{y}) \mathrm{d} S_{y} \\
& \left(\mathcal{D}^{j} v\right)(\boldsymbol{x})=\int_{\partial D_{j}} \operatorname{sgn}_{j}(\boldsymbol{y}) \frac{\partial G_{j}^{\mathrm{p}}(\boldsymbol{x}-\boldsymbol{y})}{\partial n_{y}} v(\boldsymbol{y}) \mathrm{d} S_{y}
\end{aligned}
$$

and $G_{j}^{\mathrm{p}}$ is the periodic Green function defined by

$$
\begin{aligned}
G_{j}^{\mathrm{p}}(\boldsymbol{x}-\boldsymbol{y}) & =\sum_{\boldsymbol{\xi} \in \mathcal{L}} \mathrm{e}^{\mathrm{i} \beta \cdot \boldsymbol{\xi}} G_{j}(\boldsymbol{x}-\boldsymbol{y}-\boldsymbol{\xi}) \\
G_{j}(\boldsymbol{x}-\boldsymbol{y}) & =\frac{\mathrm{e}^{\mathrm{i} k_{j}|\boldsymbol{x}-\boldsymbol{y}|}}{4 \pi|\boldsymbol{x}-\boldsymbol{y}|}
\end{aligned}
$$

with $\mathcal{L}$ being the lattice points defined by $\mathcal{L}=\left\{\left(0, \xi_{2}, \xi_{3}\right) \mid \xi_{2}=p L, \xi_{3}=\right.$ $q L, p, q \in \mathbb{Z}\}$. Also, $q_{j}$ is a function on $\partial D_{j}$ defined by

$$
q_{j}=\frac{1}{\varepsilon_{j}} \frac{\partial u_{j}}{\partial n}
$$


and $\operatorname{sgn}_{j}(\boldsymbol{x})$ is the signature function whose value is 1 when the normal vector $\boldsymbol{n}$ on $\boldsymbol{x} \in \partial D_{j}$ is inward from $D_{j},-1$ when it is outward and 0 when $\boldsymbol{x} \notin \partial D_{j}$.

From the representation in (8) one obtains

$$
\begin{aligned}
& \frac{u_{j}}{2}(\boldsymbol{x})+\left\{\varepsilon_{j} \mathcal{S}^{j}\left(\operatorname{sgn}_{j} q_{j}\right)-\mathcal{D}^{j} u_{j}\right\}(\boldsymbol{x})=\delta_{j 1} u^{\mathrm{I}}(\boldsymbol{x}) \\
& \frac{\operatorname{sgn}_{j} q_{j}}{2}(\boldsymbol{x})+\left\{\mathcal{D}^{* j}\left(\operatorname{sgn}_{j} q_{j}\right)-\frac{1}{\varepsilon_{j}} \mathcal{N}^{j} u_{j}\right\}(\boldsymbol{x})=\delta_{j 1} \operatorname{sgn}_{1}(\boldsymbol{x}) \frac{1}{\varepsilon_{1}} \frac{\partial u^{\mathrm{I}}}{\partial \boldsymbol{n}}(\boldsymbol{x})
\end{aligned}
$$

for $\boldsymbol{x} \in \partial D_{j}$, where

$$
\begin{aligned}
\left(\mathcal{D}^{* j} v\right)(\boldsymbol{x}) & =\int_{\partial D_{j}} \operatorname{sgn}_{j}(\boldsymbol{x}) \frac{\partial G_{j}^{\mathrm{p}}(\boldsymbol{x}-\boldsymbol{y})}{\partial n_{x}} v(\boldsymbol{y}) \mathrm{d} S_{y} \\
\left(\mathcal{N}^{j} v\right)(\boldsymbol{x}) & =\int_{\partial D_{j}} \operatorname{sgn}_{j}(\boldsymbol{x}) \operatorname{sgn}_{j}(\boldsymbol{y}) \frac{\partial^{2} G_{j}^{\mathrm{p}}(\boldsymbol{x}-\boldsymbol{y})}{\partial n_{x} \partial n_{y}} v(\boldsymbol{y}) \mathrm{d} S_{y} .
\end{aligned}
$$

The singular integral in (14) is evaluated in the sense of finite part if necessary. From (1), (2), (11) and (12), we obtain the following boundary integral equations for $\boldsymbol{x} \in \partial D$ :

$$
\begin{aligned}
& \sum_{j} \operatorname{sgn}_{j}(\boldsymbol{x})\left\{\varepsilon_{j} \mathcal{S}^{j}\left(\operatorname{sgn}_{j} q\right)-\mathcal{D}^{j} u\right\}(\boldsymbol{x})=\operatorname{sgn}_{1}(\boldsymbol{x}) u^{\mathrm{I}}(\boldsymbol{x}) \\
& \sum_{j}\left\{\mathcal{D}^{* j}\left(\operatorname{sgn}_{j} q\right)-\frac{1}{\varepsilon_{j}} \mathcal{N}^{j} u\right\}(\boldsymbol{x})=\operatorname{sgn}_{1}(\boldsymbol{x}) \frac{1}{\varepsilon_{1}} \frac{\partial u^{\mathrm{I}}}{\partial \boldsymbol{n}}(\boldsymbol{x})
\end{aligned}
$$

where $\partial D=\cup_{j} \partial D_{j}$. The unknown functions $u$ and $q$ are defined by

$$
u=u_{i}=u_{j}, q=\frac{1}{\varepsilon_{i}} \frac{\partial u_{i}}{\partial n}=\frac{1}{\varepsilon_{j}} \frac{\partial u_{j}}{\partial n} \quad \text { on } \partial D_{i} \cup \partial D_{j} .
$$

Though the left-hand sides of (15) and (16) appear to be summed up for all the subdomains, they are actually sums over the subdomains whose boundaries include $\boldsymbol{x}$. Also, note that the right-hand sides of equations (15) and (16) are equal to 0 unless $\boldsymbol{x} \in \partial D_{1}$ holds.

We note that integral equations in (15) and (16) include sums of integral operators in (9), (10), (13) and (14). These equations are considered to be the Helmholtz counterpart of the PMCHWT formulation in Maxwell's equations $([3,13]$, for example), which is widely used in scattering problems for dielectric objects. It is also possible to derive other integral equations which 
are Fredholm integral equations of the 2nd kind including differences of integral operators in (9) (10), (13) and (14) (See [4], for example). Comparison of numerical performances of these formulations will be an interesting future research subject. We shall, however, concentrate on the equations in (15) and (16) in the present paper.

\subsection{Discretisation of boundary integral equations}

In this paper, we discretise (15) and (16) by using piecewise linear basis functions and the Galerkin method. We discretise the boundary of the domains by a triangular mesh and define $N_{h}$ as the number of the vertices of the triangles and $t_{n}(\boldsymbol{x})$ as the piecewise linear basis whose value is one at the $n$th vertex of the mesh. By discretising (15) and (16) and expanding the unknown functions $u$ and $q$ with $t_{n}(\boldsymbol{x})$, we obtain the following equations:

$$
\begin{aligned}
& \sum_{m=1}^{N_{h}}\left(S_{n m} q_{m}-D_{n m} u_{m}\right)=u_{n}^{\mathrm{I}} \\
& \sum_{m=1}^{N_{h}}\left(D_{n m}^{*} q_{m}-N_{n m} u_{m}\right)=q_{n}^{\mathrm{I}}
\end{aligned}
$$

where $u_{n}^{\mathrm{I}}$ and $q_{n}^{\mathrm{I}}$ are defined by

$$
u_{n}^{\mathrm{I}}=\int_{\partial D_{1}} t_{n}(\boldsymbol{x}) \operatorname{sgn}_{1}(\boldsymbol{x}) u^{\mathrm{I}}(\boldsymbol{x}) \mathrm{d} S, \quad q_{n}^{\mathrm{I}}=\int_{\partial D_{1}} \frac{1}{\varepsilon_{1}} t_{n}(\boldsymbol{x}) \frac{\partial u^{\mathrm{I}}(\boldsymbol{x})}{\partial \boldsymbol{n}} \mathrm{d} S,
$$

$S_{n m}, D_{n m}, D_{n m}^{*}$ and $N_{n m}$ are defined by

$$
\begin{aligned}
& S_{n m}=\sum_{j} \varepsilon_{j} S_{n m}^{j} \quad D_{n m}=\sum_{j} D_{n m}^{j} \\
& D_{n m}^{*}=\sum_{j} D_{n m}^{* j} \quad N_{n m}=\sum_{j} \frac{1}{\varepsilon_{j}} N_{n m}^{j},
\end{aligned}
$$


and $S_{n m}^{j}, D_{n m}^{j}, D_{n m}^{* j}$ and $N_{n m}^{j}$ are defined by

$$
\begin{aligned}
S_{n m}^{j} & =\int_{\partial D_{j}} t_{n}(\boldsymbol{x}) \operatorname{sgn}_{j}(\boldsymbol{x})\left(\mathcal{S}^{j}\left(\operatorname{sgn}_{j} t_{m}\right)\right)(\boldsymbol{x}) \mathrm{d} S_{x} \\
D_{n m}^{j} & =\int_{\partial D_{j}} t_{n}(\boldsymbol{x}) \operatorname{sgn}_{j}(\boldsymbol{x})\left(\mathcal{D}^{j} t_{m}\right)(\boldsymbol{y}) \mathrm{d} S_{x} \\
D_{n m}^{* j} & =\int_{\partial D_{j}} t_{n}(\boldsymbol{x})\left(\mathcal{D}^{* j}\left(\operatorname{sgn}_{j} t_{m}\right)\right)(\boldsymbol{x}) \mathrm{d} S_{x} \\
N_{n m}^{j} & =\int_{\partial D_{j}} t_{n}(\boldsymbol{x})\left(\mathcal{N}^{j} t_{m}\right)(\boldsymbol{x}) \mathrm{d} S_{x},
\end{aligned}
$$

respectively. The summations in (19) and (20) are over subdomains $D_{j}$ whose boundary contains the support of $t_{n}$.

We define $S$ as the matrix whose $(n, m)$ component is $S_{n m}$, and define $D$, $D^{*}$ and $N$ in a similar way. We also define $\boldsymbol{u}, \boldsymbol{q}, \boldsymbol{u}^{\mathrm{I}}$ and $\boldsymbol{q}^{\mathrm{I}}$ as the vectors whose $m$ th components are $u_{m}, q_{m}, u_{m}^{\mathrm{I}}$ and $q_{m}^{\mathrm{I}}$, respectively. Then, we can rewrite (17) and (18) as

$$
A \boldsymbol{x}=\boldsymbol{b}
$$

where

$$
A=\left(\begin{array}{cc}
-D & S \\
-N & D^{*}
\end{array}\right), \quad \boldsymbol{x}=\left(\begin{array}{c}
\boldsymbol{u} \\
\boldsymbol{q}
\end{array}\right), \quad \boldsymbol{b}=\left(\begin{array}{c}
\boldsymbol{u}^{\mathrm{I}} \\
\boldsymbol{q}^{\mathrm{I}}
\end{array}\right)
$$

\subsection{Periodic fast multipole method}

The periodic fast multipole method (periodic FMM) is an algorithm which rapidly calculates the left-hand sides of (15) and (16) by collectively dealing with effects from the part of the boundary far from the point $\boldsymbol{x}$ with the help of the multipole expansions. Effects from the part of the boundary near $\boldsymbol{x}$, however, are evaluated directly with the conventional BEM. In this paper, we do not elaborate on the detail of the periodic FMM. For more information, the reader is referred to Otani and Nishimura $[3,15]$.

\section{Preconditionings based on Calderon's formulae}

In this section, we consider preconditionings based on Calderon's formulae. For simplicity, we assume throughout this section that the domain $D$ consists of two subdomains $D_{1}$ and $D_{2}$, and the direction of the normal vector on boundary is fixed outward (Fig. 2). 


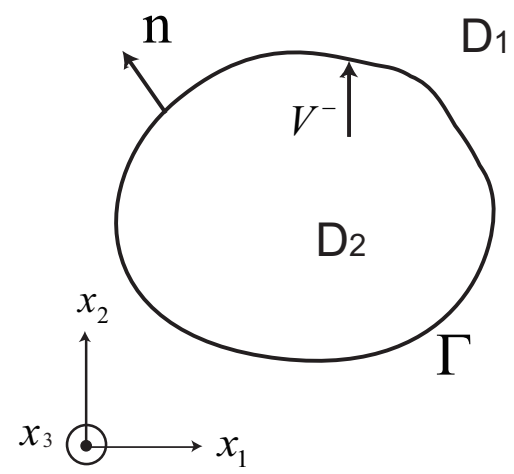

Figure 2: Domain $D_{j}(j=1,2)$ in $\mathbb{R}^{3}$.

3.1. Calderon's formulae for periodic boundary value problems

We define a function $V$ by

$V(\boldsymbol{x})=\int_{\partial D_{j}} G_{j}^{\mathrm{p}}(\boldsymbol{x}-\boldsymbol{y}) \psi(\boldsymbol{y}) \mathrm{d} S_{y}-\int_{\partial D_{j}} \frac{\partial G_{j}^{\mathrm{p}}(\boldsymbol{x}-\boldsymbol{y})}{\partial \boldsymbol{n}_{y}} \phi(\boldsymbol{y}) \mathrm{d} S_{y} \quad$ in $D_{j}, \quad j=1,2$

where $\psi$ and $\phi$ are arbitrary functions defined on the boundary $\partial D_{j}$. Then, $V$ satisfies following relations for $j=1,2$ :

$$
\begin{aligned}
\frac{V^{-}}{2} & =\int_{\Gamma} G_{j}^{p}(\boldsymbol{x}-\boldsymbol{y}) \frac{\partial V^{-}}{\partial \boldsymbol{n}} \mathrm{d} S-\int_{\Gamma} \frac{\partial G_{j}^{p}(\boldsymbol{x}-\boldsymbol{y})}{\partial \boldsymbol{n}_{y}} V^{-} \mathrm{d} S \\
\frac{1}{2} \frac{\partial V^{-}}{\partial \boldsymbol{n}} & =\int_{\Gamma} \frac{\partial G_{j}^{p}(\boldsymbol{x}-\boldsymbol{y})}{\partial \boldsymbol{n}_{x}} \frac{\partial V^{-}}{\partial \boldsymbol{n}} \mathrm{d} S-\int_{\Gamma} \frac{\partial^{2} G_{j}^{p}}{\partial \boldsymbol{n}_{x} \partial \boldsymbol{n}_{y}} V^{-} \mathrm{d} S \\
V^{-} & =\mathcal{S}^{j} \psi+\frac{\phi}{2}-\mathcal{D}^{j} \phi \\
\frac{\partial V^{-}}{\partial n} & =\frac{\psi}{2}+\mathcal{D}^{* j} \psi-\mathcal{N}^{j} \phi
\end{aligned}
$$

where $V^{-}(\boldsymbol{x})$ is the limit value of $V(\boldsymbol{x})$ from $D_{2}$ to $\Gamma$ and $\frac{\partial V^{-}}{\partial n}$ is the limit value of the normal derivative of $V(\boldsymbol{x})$ from $D_{2}$ to $\Gamma$.

From (27), (28), (29) and (30), we obtain the Calderon formulae for 
periodic problems:

$$
\begin{aligned}
\frac{\mathcal{I}}{4} & =-\mathcal{S}^{j} \mathcal{N}^{j}+\mathcal{D}^{j} \mathcal{D}^{j} \\
0 & =\mathcal{S}^{j} \mathcal{D}^{* j}-\mathcal{D}^{j} \mathcal{S}^{j} \\
\frac{\mathcal{I}}{4} & =\mathcal{D}^{* j} \mathcal{D}^{* j}-\mathcal{N}^{j} \mathcal{S}^{j} \\
0 & =-\mathcal{D}^{* j} \mathcal{N}^{j}+\mathcal{N}^{j} \mathcal{D}^{j}
\end{aligned}
$$

where $\mathcal{I}$ is an identity operator.

\subsection{Preconditionings based on Calderon's formulae with collocation}

We now summarise results from [14] where preconditioning methods for the linear equations obtained from (15) and (16) with collocation are discussed.

To start with, we note that (31), (32), (33) and (34) can be expressed as

$$
\left(\begin{array}{cc}
-\mathcal{D}^{j} & \mathcal{S}^{j} \\
-\mathcal{N}^{j} & \mathcal{D}^{* j}
\end{array}\right)\left(\begin{array}{cc}
-\mathcal{D}^{j} & \mathcal{S}^{j} \\
-\mathcal{N}^{j} & \mathcal{D}^{* j}
\end{array}\right)=\left(\begin{array}{cc}
\frac{\mathcal{I}}{4} & 0 \\
0 & \frac{\mathcal{I}}{4}
\end{array}\right)
$$

This equation means that the integral operator

$$
\left(\begin{array}{ll}
-\mathcal{D}^{j} & \mathcal{S}^{j} \\
-\mathcal{N}^{j} & \mathcal{D}^{* j}
\end{array}\right)
$$

is the inverse of itself up to the factor of $1 / 4$. In addition, it is known that the following relationship holds

$$
\left(\begin{array}{cc}
-\sum_{j} \mathcal{D}^{j} & \sum_{j} \varepsilon_{j} \mathcal{S}^{j} \\
-\sum_{j} \frac{1}{\varepsilon_{j}} \mathcal{N}^{j} & \sum_{j} \mathcal{D}^{* j}
\end{array}\right)\left(\begin{array}{cc}
-\sum_{j} \mathcal{D}^{j} & \sum_{j} \varepsilon_{j} \mathcal{S}^{j} \\
-\sum_{j} \frac{1}{\varepsilon_{j}} \mathcal{N}^{j} & \sum_{j} \mathcal{D}^{* j}
\end{array}\right)=\left(\begin{array}{cc}
\mathcal{I} & 0 \\
0 & \mathcal{I}
\end{array}\right)+\left(\begin{array}{ll}
\mathcal{K}_{1} & \mathcal{K}_{2} \\
\mathcal{K}_{3} & \mathcal{K}_{4}
\end{array}\right)
$$

where $\mathcal{K}_{1}, \mathcal{K}_{2}, \mathcal{K}_{3}$ and $\mathcal{K}_{4}$ are compact operators[10]. Namely, $\mathcal{A}^{2}$ is a compact perturbation of the identity where

$$
\mathcal{A}=\left(\begin{array}{cc}
-\sum_{j} \mathcal{D}^{j} & \sum_{j} \varepsilon_{j} \mathcal{S}^{j} \\
-\sum_{j} \frac{1}{\varepsilon_{j}} \mathcal{N}^{j} & \sum_{j} \mathcal{D}^{* j}
\end{array}\right)
$$

As a result we see that the linear equation

$$
\tilde{A} \boldsymbol{x}=\tilde{\boldsymbol{b}}
$$


obtained by discretising (17) and (18) with collocation (which is different from the Galerkin equation in (25)) can be preconditioned by $\tilde{A}$ itself (i.e., we can use $\tilde{A}^{-1}$ as the preconditioner), where $\tilde{A}$ is the collocation discretisation of $\mathcal{A}$. Notice that solving the linear equation in (36) with the (right) preconditioner $\tilde{A}^{-1}$ means solving $\tilde{A}^{2} \boldsymbol{y}=\boldsymbol{b}$ and $\boldsymbol{x}=\tilde{A} \boldsymbol{y}$ instead of solving (36) according to the standard definition of preconditioners[7], which is somewhat confusing in the present context. Therefore, there is no matrix inversion involved in using $\tilde{A}^{-1}$ as the preconditioner.

We can show that the solution of (36) converges faster (with equal or less number of matrix-vector product operations, to be precise) with no preconditioner than with $\tilde{A}^{-1}$ as the right preconditioner if we use GMRES as the iterative solver and have enough memory so that we do not need to restart GMRES. Indeed, if we solve (36) with the preconditioner $\tilde{A}^{-1}$, it takes two matrix-vector products in every iteration of GMRES. However, if we solve (36) with no preconditioner, we need to calculate only one matrix-vector product in every iteration. Therefore, the computational time of GMRES with no preconditioner for $2 n$ iterations is nearly equal to that of GMRES with preconditioner $\tilde{A}^{-1}$ for $n$ iterations since the matrix-vector product takes by far the largest part in the whole computational time. It is known that GMRES after $n$ iterations finds the solution which minimizes the residual of the linear equation in (36) in the Krylov subspace. GMRES after $n$ iterations with the preconditioner $\tilde{A}^{-1}$ finds the solution in the following affine space:

$$
\boldsymbol{x}_{0}+\left\{\tilde{A} \boldsymbol{v}, \tilde{A}^{3} \boldsymbol{v}, \tilde{A}^{5} \boldsymbol{v}, \cdots, \tilde{A}^{2 n-1} \boldsymbol{v}\right\}
$$

while GMRES after $2 n$ iterations with no preconditioner finds the solution in the affine space given by:

$$
\boldsymbol{x}_{0}+\left\{\boldsymbol{v}, \tilde{A} \boldsymbol{v}, \tilde{A}^{2} \boldsymbol{v}, \cdots, \tilde{A}^{2 n-1} \boldsymbol{v}\right\}
$$

where $\boldsymbol{x}_{0}$ is the initial guess and $\boldsymbol{v}$ is the initial residual. Therefore, GMRES with no preconditioner converges faster in terms of the computational time than GMRES with the preconditioner $\tilde{A}^{-1}$ because the space (38) contains (37).

We verified this fact for Helmholtz' equation in 2D numerically in [14].

\subsection{Preconditionings based on Calderon's formulae with the Galerkin method}

In this subsection, we consider similar Calderon preconditionings with the Galerkin discretisation. 
We first note that we cannot simply apply the preconditioning scheme stated above in the case of the Galerkin discretisation. Indeed, a relation $\eta^{j}=\mathcal{S}^{j} \zeta$ with

$$
\zeta(x) \approx \sum_{n=1}^{N_{h}} \zeta_{n} t_{n}(x), \quad \eta^{j}(x) \approx \sum_{n=1}^{N_{h}} \eta_{n}^{j} t_{n}(x)
$$

is easily seen to be discretised into

$$
\sum_{m=1}^{N_{h}} S_{n m}^{j} \zeta_{m}=\sum_{m=1}^{N_{h}} \eta_{m}^{j} T_{n m}^{j}
$$

where

$$
T_{n m}^{j}=\int_{\partial D_{j}} t_{n}(x) t_{m}(x) \mathrm{d} S_{x}
$$

is the Gram matrix. Summing up (39) with respect to the index $j$, we obtain

$$
S \boldsymbol{\zeta} \approx T^{\prime} \boldsymbol{\eta}
$$

where $\boldsymbol{\zeta}$ and $\boldsymbol{\eta}$ are vectors whose $n$th component are $\zeta_{n}$ and $\sum_{j} \eta_{n}^{j}$ and

$$
T_{m n}^{\prime}=\int_{\partial D} t_{m}(\boldsymbol{x}) t_{n}(\boldsymbol{x}) \mathrm{d} S_{\boldsymbol{x}}
$$

In the present context, we have $T_{m n}^{\prime}=T_{m n}^{1}=T_{m n}^{2}$. Since $T^{\prime}$ is regular, we obtain

$$
\boldsymbol{\eta} \approx T^{-1} S \zeta
$$

which implies that a product $\mathcal{D S} \boldsymbol{\zeta}$ is discretised into

$$
T^{\prime-1} D T^{-1} S \zeta \text {. }
$$

From (25), (35) and (40), we see that the coefficient matrix in (25) satisfies

$$
T^{-1} A T^{-1} A \approx I+K^{\prime}
$$

where $K^{\prime}$ is a matrix which is obtained by discretising a compact operator and $T$ is given by

$$
\left(\begin{array}{cc}
T^{\prime} & 0 \\
0 & T^{\prime}
\end{array}\right)
$$


Similarly, one has

$$
A T^{-1} A T^{-1} \approx I+K
$$

where $K=T K^{\prime} T^{-1}$. Consequently, we can precondition (25) using the matrix $T A^{-1} T$ as a right preconditioner if we use Galerkin's method.

Furthermore, (41) implies that we can use $T$, instead of $T A^{-1} T$, as a preconditioner for (25) because the square of the matrix $A T^{-1}$ is approximately equal to $I+K$. Indeed, we can use the same argument as has been used in section 3.2 to show that (25) preconditioned with $T$ converges faster than that using $T A^{-1} T$, as far as the computational time is concerned.

Note that this preconditioning with $T$ is possible because we have ordered the equations and unknowns as in (26). If we use different orderings, however, we cannot expect $T$ to work as a preconditioner. For example, the ordering used by Antoine and Boubendir converts (25) into

$$
\hat{A} \hat{\boldsymbol{x}}=\hat{\boldsymbol{b}}
$$

where

$$
\hat{A}=\left(\begin{array}{cc}
S & -D \\
-D^{*} & N
\end{array}\right) \quad \hat{\boldsymbol{x}}=\left(\begin{array}{c}
\boldsymbol{q} \\
\boldsymbol{u}
\end{array}\right) \quad \hat{\boldsymbol{b}}=\left(\begin{array}{c}
\boldsymbol{u}^{\mathrm{I}} \\
-\boldsymbol{q}^{\mathrm{I}}
\end{array}\right)
$$

Calderon's formulae tell that equation (42) can be right-preconditioned by

$$
T\left(\begin{array}{cc}
-N & -D^{*} \\
-D & -S
\end{array}\right)^{-1} T
$$

or left-preconditioned similarly, but (42) cannot be preconditioned by $T$ because the central matrix in (43) has an ordering different from that for $\hat{A}$. This difference between $A$ and $\hat{A}$ is understood from the spectral properties of the corresponding operators. Indeed, one shows that the eigenvalues of $\mathcal{A}$ accumulate at 1 , while those of $\hat{\mathcal{A}}$ (continuous counterpart of $\hat{A}$ obtained by arranging the components of $\mathcal{A}$ appropriately) accumulate either at 0 or at $\infty$, thus indicating a worse conditioning of $\hat{A}$ for a finer mesh.

We remark that the relevance of the Gram matrix in the Calderon preconditioning is known[8], but its effectiveness as a preconditioner has not been pointed out so far, to the best of the present authors' knowledge. 


\subsection{Calculation of matrix $T$}

The matrix $T$ is symmetric, sparse, diagonally dominant and depends only on the mesh on the boundary. Hence, we can compute the product of $T$ and an arbitrary vector with $O\left(N_{h}\right)$ computational time. This suggests that the inverse of $T$ can be calculated efficiently with Krylov subspace iteration methods. We therefore use "flexible" iterative methods [7] which enable us to use different preconditioners for different iteration steps, thus making the use of iterative methods for the inversion of $T$ possible. In this method, the computational time of $T^{-1}$ is $O\left(N_{h}\right)$ if the number of iterations is independent of $N_{h}$.

\section{Numerical examples}

In this study, we compare the efficiency of the following 6 approaches to solve the system of integral equations in (15) and (16). The first 4 are based on (25) and the last 2 are related to (42).

1. The first approach solves (25) with FGMRES (flexible GMRES, see Sec. 3.4) with $T$ as the right preconditioner.

2. The second approach solves (25) with FGMRES with $T A^{-1} T$ as the right preconditioner.

3. The third approach also solves (25) with FGMRES and a right preconditioner which is the part of the matrix computed directly in the FMM algorithm, i.e., the part of the coefficient matrix representing the interactions between neighbour leaves [3].

4. The fourth approach solves the unpreconditioned equation (25) as is with GMRES.

5. The fifth approach solves (42) with FGMRES using the part of the matrix computed directly in the FMM algorithm as the right preconditioner.

6. The sixth approach solves (42) as is with GMRES.

For the evaluation of integrals in (21), (22), (23) and (24) needed in calculating the preconditioner in the approaches 3 and 5, we combine an analytical integration for the Laplace part $(1 /(4 \pi|\boldsymbol{x}-\boldsymbol{y}|))$ and a numerical integration for the rest (i.e., $G(\boldsymbol{x}-\boldsymbol{y})-1 /(4 \pi|\boldsymbol{x}-\boldsymbol{y}|))$ in the evaluation of (9), (10), (13) and (14). The outer integrals in (21), (22), (23) and (24) are evaluated numerically. Although it is possible to solve (42) with the preconditioning based 
on Calderon's formulae, we did not test this method because this approach is equivalent to the 2 nd approach.

In the numerical examples to follow, we use the low frequency FMM taking the periodicity into account with the help of the periodic M2L formula proposed in Otani and Nishimura[3]. We use the Fujitsu HX600 cluster (with AMD Opteron CPUs) of the data processing center of Kyoto University for the calculation with minimum shared memory parallelisation.

\subsection{Verification}

We first verify our approach by solving problems with known analytical solutions. We consider the model with two layers of spheres shown in Fig. 3. The unit domain $D$ has two spheres with the radius of 0.2 whose centres are 0.5 apart (Fig. $3 \mathrm{~b})$ ). We denote the domain outside of the spheres by $D_{1}$ and the domain in the inside of the spheres by $D_{2}$, respectively. The period $L$ is 1 in both $x_{2}$ and $x_{3}$ directions and the number of elements is 316848 . We have intentionally made the mesh very fine so that we can test the performance of the proposed approaches in relatively large problems.
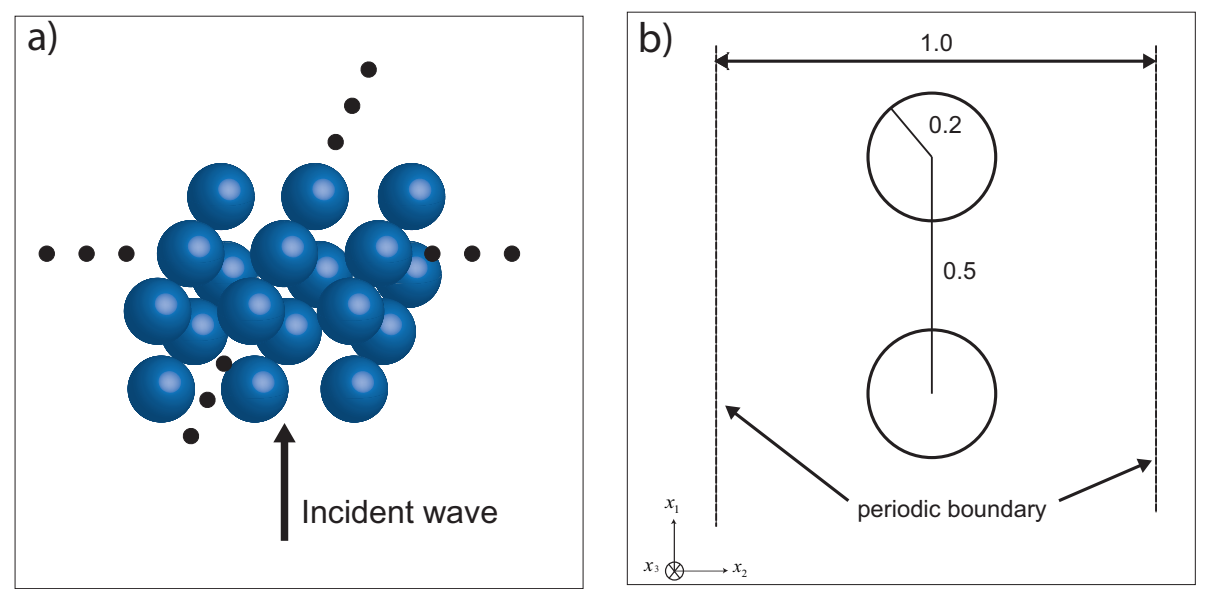

Figure 3: The model with two layers of spheres. a) The shape of the domains b) The unit domain

If $\varepsilon_{j}=1$ and $k_{j}=k$ ( $k$ is a constant) for $j=1,2$, the analytical solution of the problem is exactly (and non-trivially) equal to the incident wave. We verify the accuracy of the methods by comparing the analytical solution and the numerical solutions for this choice of $\varepsilon_{j}(j=1,2)$. The wave number 
is set equal to $k_{j}=6(j=1,2)$. The incident wave is the plane wave in (7) with $\beta_{2}=1$ and $\beta_{3}=0$. As a solver of linear equations, we use GMRES or flexible GMRES with the error tolerance of $10^{-4}$ for the outer GMRES. For the FGMRES, the inner iterative solver is also GMRES. In approaches 1 and 2, we set the error tolerance for the inversion of $T$ in the inner GMRES to be $10^{-15}$ and the maximum number of iterations to be 10. We stop the inner iteration when either of these conditions is satisfied. We selected this extremely small error tolerance in order to calculate $T^{-1}$ as precisely as possible so that we can fully utilise the effect of Calderon's formulae. As a matter of fact, the convergence of this iteration is very fast and usually reaches the $10^{-15}$ threshold within 10 iterations. This is because $T$ is well-conditioned. For approaches 3 and 5 the error tolerance of the inner GMRES is set to be $10^{-3}$ with the maximum number of iterations being 10 . This is because we just need a rough approximation of the inverse of the preconditioner since the main purpose of this preconditioning is to scale the coefficient matrix properly.

We now show the relative errors $u_{\text {err }}$ and $q_{\text {err }}$ defined by

$$
\begin{aligned}
u_{\mathrm{err}} & =\frac{\int_{\partial D}\left|u_{\mathrm{cal}}-u_{\mathrm{ana}}\right| \mathrm{d} S}{\int_{\partial D}\left|u_{\text {ana }}\right| \mathrm{d} S} \\
q_{\mathrm{err}} & =\frac{\int_{\partial D}\left|q_{\mathrm{cal}}-q_{\mathrm{ana}}\right| \mathrm{d} S}{\int_{\partial D}\left|q_{\mathrm{ana}}\right| \mathrm{d} S}
\end{aligned}
$$

for each of the preconditioning approaches in Table 1 , where $u_{\text {cal }}$ and $q_{\text {cal }}$ are the numerical solutions, $u_{\text {ana }}$ and $q_{\text {ana }}$ are the analytical solutions and $\partial D$ is the boundary of all the subdomains.

\begin{tabular}{|c|c|c|c|c|}
\hline name & Coefficient matrix & Preconditioning & $u_{e r r}$ & $\partial u_{\mathrm{err}} / \partial \boldsymbol{n}$ \\
\hline approach 1 & \multirow{4}{*}{$A$} & Calderon with $T$ & $5.50 \times 10^{-5}$ & $7.26 \times 10^{-3}$ \\
\hline approach 2 & & Calderon with $T A^{-1} T$ & $5.73 \times 10^{-5}$ & $7.06 \times 10^{-3}$ \\
\hline approach 3 & & Direct & $5.74 \times 10^{-5}$ & $8.21 \times 10^{-3}$ \\
\hline approach 4 & & No preconditioning & $5.74 \times 10^{-5}$ & $7.20 \times 10^{-3}$ \\
\hline approach 5 & \multirow{2}{*}{$\hat{A}$} & Direct & $6.51 \times 10^{-5}$ & $1.16 \times 10^{-2}$ \\
\hline approach 6 & & No preconditioning & $7.11 \times 10^{-5}$ & $1.27 \times 10^{-2}$ \\
\hline
\end{tabular}

Table 1: Relative error. 
From Table 1, we can say that all the preconditioning approaches considered give equally accurate numerical results.

\subsection{Computational time}

We now consider the domain shown in Fig. 3 with $\varepsilon_{1}=1$, and investigate the number of iterations of GMRES and computational time for various values of $\varepsilon_{2} \leq 1$. For such $\varepsilon_{2}$ we expect no anomalous behaviour of the solution [16] except possibly at Rayleigh's anomalies. Figs. 4 a) and b) show the number of iterations and computational time for each of the preconditioning approaches, respectively. The horizontal axis gives the value of $\varepsilon_{2}$.

a)

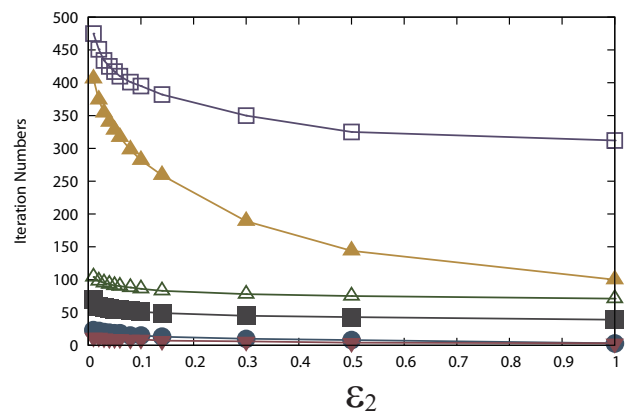

C)

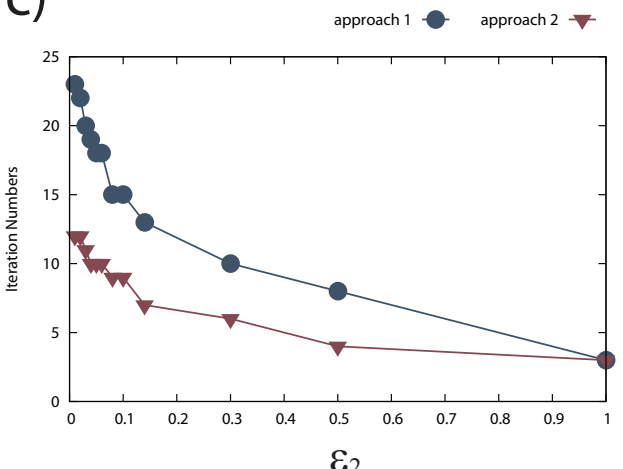

b)

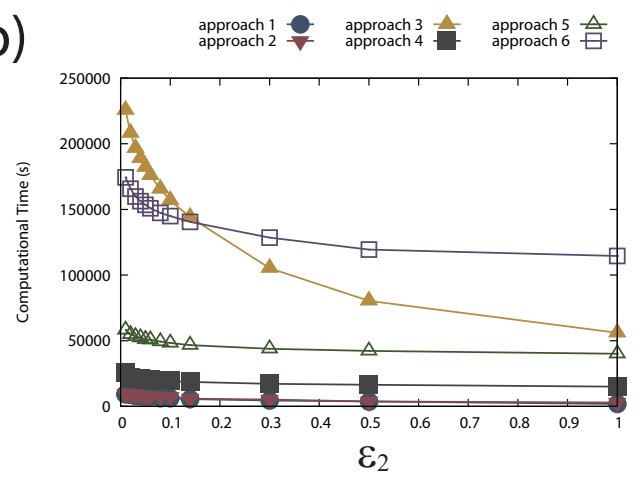

d)

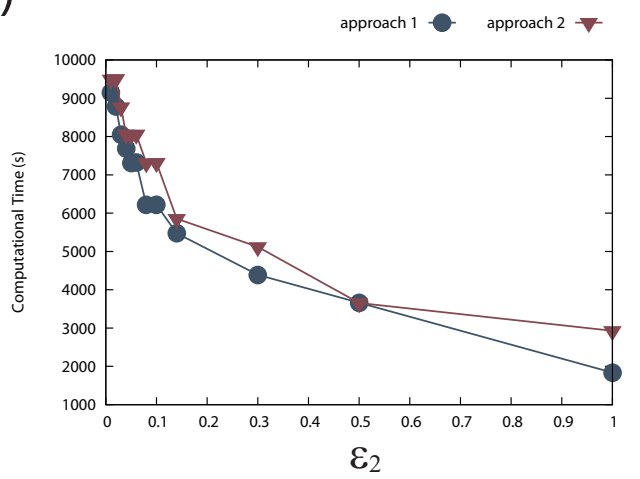

Figure 4: The number of iterations and the computational time of GMRES and FGMRES for the model with two layers of spheres. a) The number of iterations for the six approaches. b) The computational time for the six approaches. c) The number of iterations for the approaches 1 and 2. d) The computational time for the approaches 1 and 2.

We first note that the preconditioning approaches based on Calderon's 
formulae, i.e., approaches 1 and 2 are faster than other approaches even when $\varepsilon_{2}$ is much different from $\varepsilon_{1}$. To compare these two preconditionings more closely, we plotted the iteration number and the computational time of these two approaches for the same problem in Figs. 4 c) and d), respectively.

We expect that the computational time for the approach 1 is shorter than that for the approach 2, while the iteration number of the approach 1 is more than that of the approach 2. The reason is similar to the collocation case. Namely, we need to calculate the products of $A$ with a vector just once in every iteration in the approach 1, while we need the same product twice in every iteration in the approach 2. Moreover, the Krylov subspace after $n$ iterations for the approach 2 is included in that of the approach 1 after $2 n$ iterations as explained in section 3.2. Since the computational time in BEM depends mainly on the number of matrix vector products, the residual with the approach 1 is expected to be smaller than that of the approach 2 with GMRES after the same amount of the computational time. We see that the results in Fig. 4 are in agreement with this expectation.

In Fig. 5, we fixed $\varepsilon_{2}=0.1$ and solved problems for various wave numbers of the incident wave $k_{1}$. Fig. 5 a) shows the iteration number for each of the 6 preconditioning approaches while Fig. 5 b) presents the corresponding computational time, respectively. We set the maximum number of iterations of outer GMRES to be 1000. As seen in Fig. 5 a) the approach 6 failed to converge when the wave number is 20,25 , and 30 . In Figs. 5 c) and d), we compare the preconditioners based on Calderon's formulae (i.e. approaches 1 and 2) in terms of the iteration number and computational time, respectively. All the results show the same tendencies as have been observed in the variable $\varepsilon_{2}$ case.

\subsection{Performance near anomalies and guided modes}

To investigate the performances of the proposed preconditioning approaches near anomalies, we consider another problem for the domains shown in Fig. 6. We denote the domain outside of the sphere by $D_{1}$ and the domain inside of the sphere by $D_{2}$. The radius of the sphere is 0.35 and the period $L$ is 1 in both $x_{2}$ and $x_{3}$ directions. We set $\varepsilon_{1}=1, \varepsilon_{2}=2.56$ and, hence, $k_{2}=k_{1} \sqrt{\varepsilon_{2}}=1.6 k_{1}$. In this problem, we expect the solution to show anomalous behaviour because $k_{1}<k_{2}$ holds. Indeed, it is known, in this case, that (see [16] for the corresponding 2D cases) the following results hold:

1. There exist any given number of guided modes (solutions to homoge- 
a)

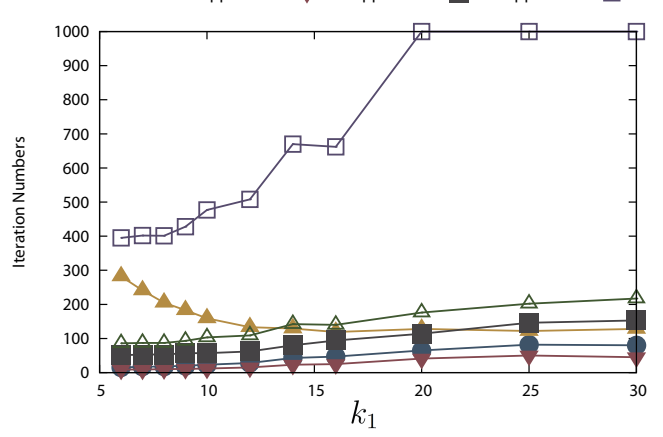

C)

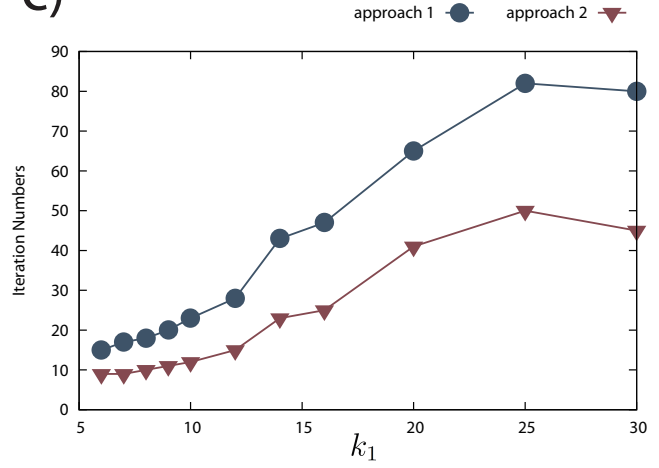

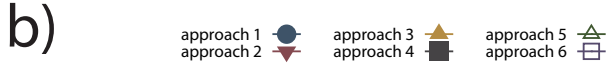

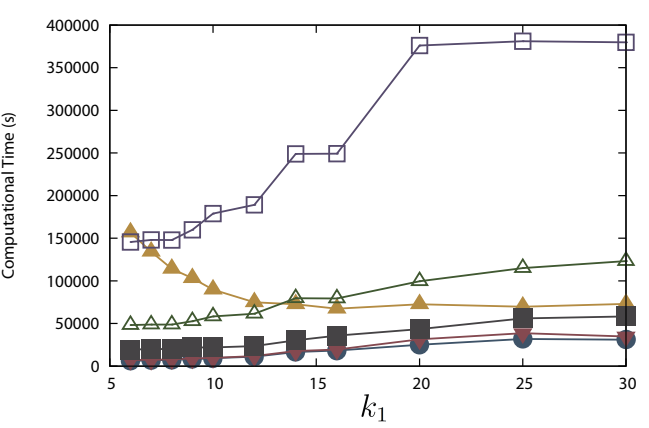

d)

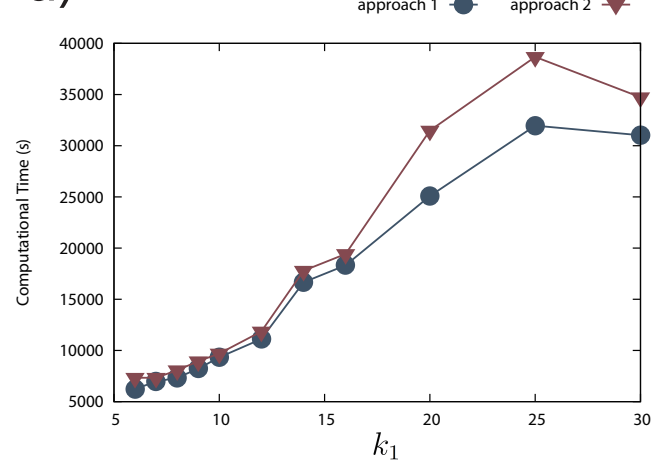

Figure 5: The number of iterations and the computational time of GMRES and FGMRES for the model with two layers of spheres. a) The number of iterations for the six approaches. b) The computational time for the six approaches. c) The number of iterations for the approach 1 and 2. d) The computational time for the approach 1 and 2 . 
neous problems) which satisfy

$$
k_{1} L<|\boldsymbol{\beta}|<k_{2} L
$$

if the contrast $\varepsilon_{2} / \varepsilon_{1}$ is sufficiently high. These guided modes are called 'robust'.

2. There exist guided modes which satisfy

$$
|\boldsymbol{\beta}|<k_{1} L<2 \pi
$$

when $\beta_{2}=0$ and the structure is symmetric with respect to the $x_{2}=0$ plane, provided the contrast $\varepsilon_{2} / \varepsilon_{1}$ is sufficiently high[17]. These guided modes are called 'embedded'.

3. The scattering problem has at least one solution when the incident wave is planar, regardless of the existence or nonexistence of the guided modes.

The frequencies where guided modes exist are called 'singular frequencies'. The $3 \mathrm{rd}$ of the above results is of interest from practical viewpoints. However, other results (2nd one in particular) are also relevant to the case of the plane wave incidence, as we shall see.

\subsubsection{Robust guided modes}

Robust guided modes can be excited easily by an incident evanescent wave given by

$$
u^{\mathrm{I}}(\boldsymbol{x})=C_{\text {inc }} \mathrm{e}^{\mathrm{i} k_{1} \boldsymbol{v} \cdot \boldsymbol{x}}
$$

$\boldsymbol{v}=\left(\mathrm{i} \sqrt{\left(\frac{\beta_{2}}{k_{1} L}\right)^{2}+\left(\frac{\beta_{3}}{k_{1} L}\right)^{2}-1}, \frac{\beta_{2}}{k_{1} L}, \frac{\beta_{3}}{k_{1} L}\right), \quad\left(\frac{\beta_{2}}{L}\right)^{2}+\left(\frac{\beta_{3}}{L}\right)^{2}-\left|k_{1}\right|^{2}>0$.

We here set $\beta_{2}=3, \beta_{3}=0$ and $C_{\text {inc }}=1$.

To examine the behaviour of the solution, we have computed the value of $|u|$ at $\boldsymbol{x} \approx(-0.35,0,0)$ using FMBEM with the preconditioning approach 1 and a 5780 element mesh, and plotted the results for various values of $k_{1}$ in Fig. 7. We see that $|u|$ diverges near $k_{1}=2.94$, indicating that the solution ceases to exist at a certain $k_{1}$ near 2.94 .

This wavenumber $(\approx 2.94)$ is considered to correspond to a guided mode. Indeed, we can obtain a rough estimate of $k_{1}$ corresponding to the singular 


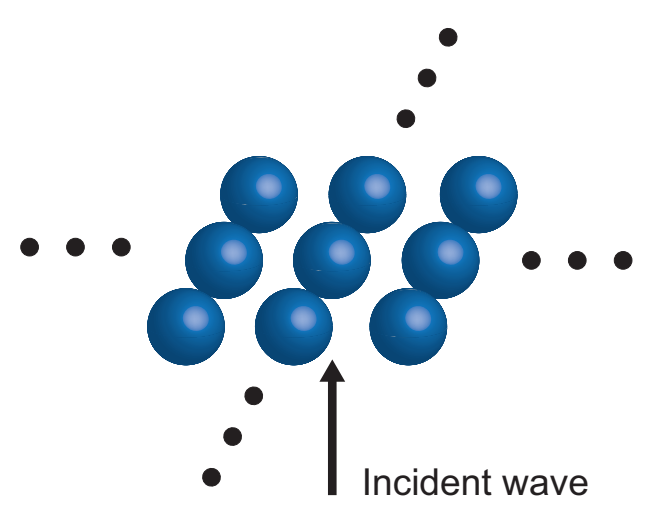

Figure 6: The model of spheres.

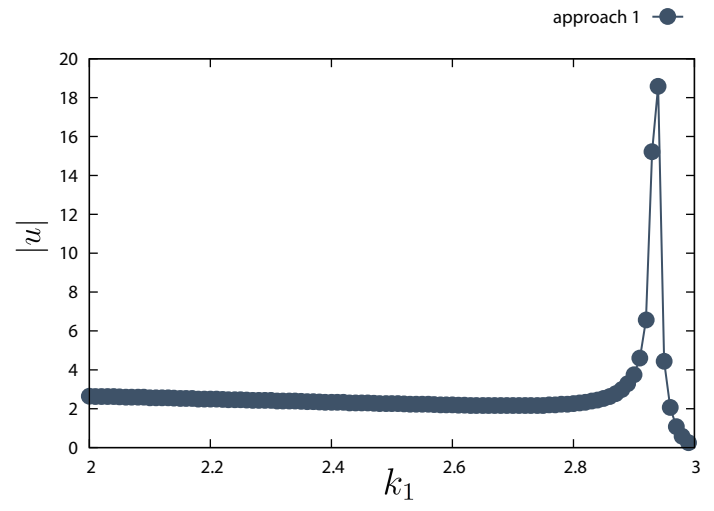

Figure 7: The value of $|u|$ at $\boldsymbol{x} \approx(-0.35,0,0)$ in the case of an incident evanescent wave. 
frequency by approximating the scatterers by an uniform layer sandwiched between homogeneous halfspaces (we call this the 3 layer model), where an analytical treatment is possible[6]. One may well assume the thickness of this layer to be 0.7 and the value of $\varepsilon$ to be a certain average of $\varepsilon_{1}$ and $\varepsilon_{2}$. If we average $\varepsilon_{1}$ and $\varepsilon_{2}$ via $(1-f) \varepsilon_{1}+f \varepsilon_{2}$ with the volume fraction of spheres in the layer denoted by $f$, we obtain $\varepsilon \approx 1.4$ which leads to $k_{1} \approx 2.90$. If we average the reciprocal of $\varepsilon$ by $\frac{1}{(1-f) / \varepsilon_{1}+f / \varepsilon_{2}}$ we obtain $\varepsilon \approx 1.185$ or $k_{1} \approx 2.97$. The observed value $k_{1}=2.94$ is located in between these numbers.

Fig. 8 show the iteration number and computational time for solving this problem with the 6 types of preconditioners. From these figures, we see that the iteration numbers for preconditioning approaches based on Calderon's formulae (approaches 1 and 2) are always lower than those for other preconditioning approaches. Also, the iteration numbers of the approaches 1 and 2 remain almost constant even near $k_{1}=2.94$ while approaches based on (42) (approaches 5 and 6) suffer from increased iteration numbers and computational times there.
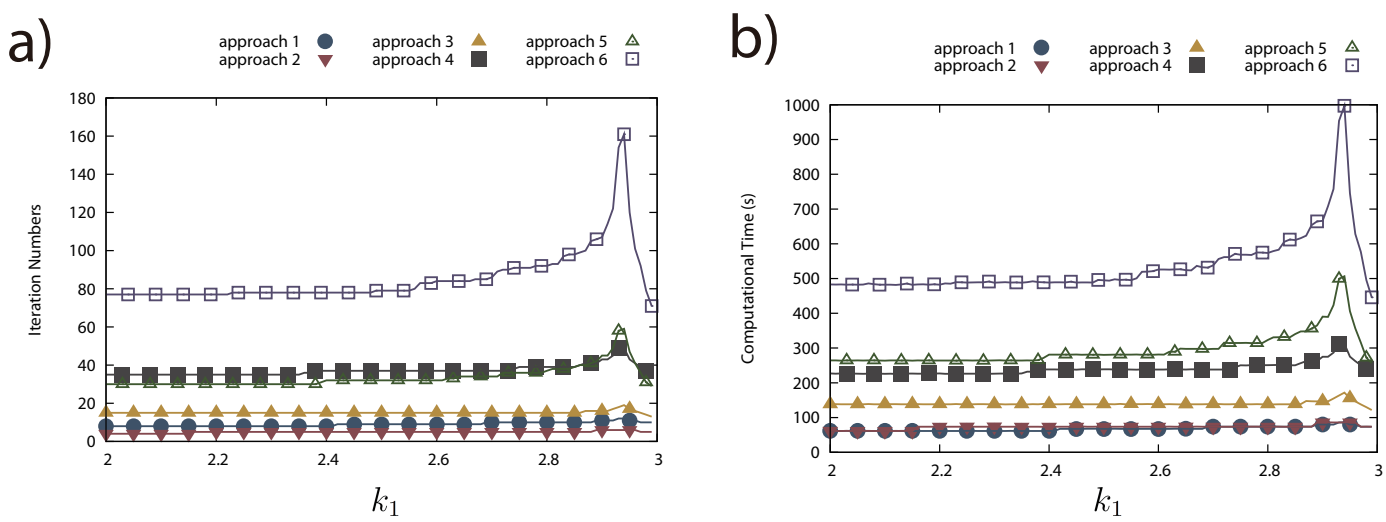

Figure 8: The number of iterations of GMRES and FGMRES for the model of spheres around a robust guided mode. a) The number of iterations b)The computational time

\subsubsection{Embedded guided modes and plane wave incidence}

We consider the same problem as in the previous example except that we take $\beta_{2}=\beta_{3}=0$. In this case, we may have embedded guided modes. As a matter of fact, the 3 layer model tells that we may expect guided modes near $k_{1} \approx 5.76(\varepsilon \approx 1.4)$ or $k_{1} \approx 6.08(\varepsilon \approx 1.185)$. 
To find these guided modes, we compute the response of this system subject to an incident wave given by

$$
\begin{gathered}
u^{\mathrm{I}}(\boldsymbol{x})=C_{\mathrm{inc}} \mathrm{e}^{\mathrm{i} k_{1} \boldsymbol{v} \cdot \boldsymbol{x}} \\
\boldsymbol{v}=\left(\mathrm{i} \sqrt{\left(\frac{\beta_{2}}{k_{1} L}+\frac{2 \pi}{k_{1}}\right)^{2}+\left(\frac{\beta_{3}}{k_{1} L}\right)^{2}-1}, \frac{\beta_{2}}{k_{1} L}+\frac{2 \pi}{k_{1}}, \frac{\beta_{3}}{k_{1} L}\right)
\end{gathered}
$$

with $\beta_{2}=0, \beta_{3}=0$ and $C_{\mathrm{inc}}=1$. This incident wave is evanescent for $k_{1}<2 \pi$. Fig. 9 shows the value of $|u|$ at $\boldsymbol{x}=(0,0.35,0)$ computed with the approach 1 . We notice in Fig. 9 that $|u|$ diverges at $k_{1}=5.89$ and 5.97, indicating the existence of two guided modes in the interval predicted by the 3 layer model. In addition to these guided modes, we recognise an anomaly at $k_{1}=5.5$ in the $k_{1}<2 \pi$ range.

We next consider the response of the same system subject to a plane incident wave given by

$$
u^{\mathrm{I}}(\boldsymbol{x})=\mathrm{e}^{\mathrm{i} k_{1} x_{1}} .
$$

Fig. 10 shows $|u|$ at $\boldsymbol{x}=(-0.35,0,0)$ vs $k_{1}$ obtained with the approach 1. As in the case of the incident evanescent wave, we see that the solution suddenly changes near $k_{1}=5.5$. Also, we can identify a Rayleigh's anomaly at $k_{1}=2 \pi$ where the slope of the solution varies rapidly. However, the solution stays smooth near $k_{1}=5.89$ and 5.97. This result reflects the fact that the solution to the scattering problem exists as long as the incident wave is planar even when guided modes exist. Fig. 11 shows the iteration number and the computational time for the incident plane wave case. We notice that conventional approaches (approaches 3 to 6) suffer from increased iteration numbers and computational time at the anomaly near $k_{1}=5.5$ as well as at the guided modes. With the Calderon preconditionings (approaches 1 and 2), however, the iteration numbers stay low even at these anomalies and guided modes. Fig. 12 shows the values of $|u|$ at $\boldsymbol{x} \approx(-0.35,0,0)$ around $k_{1}=5.89$ and 5.97 obtained with the 6 preconditioning approaches, where the incident wave is the plane wave given in (46). As in Fig. 10, the Calderon results (approaches 1 and 2) in Fig. 12 also remain smooth even near $k_{1}=5.89$ and 5.97. However, results obtained with other 4 approaches are off the solution curve by a small amount at these values of $k_{1}$, reflecting the fact that the 


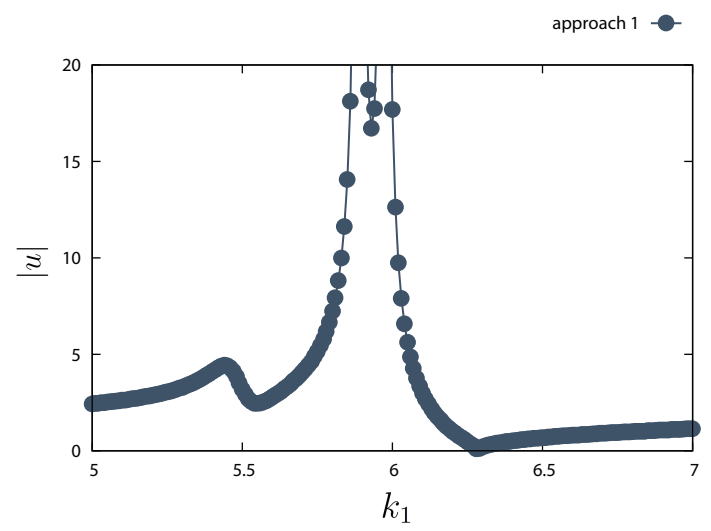

Figure 9: The value of $|u|$ at $\boldsymbol{x} \approx(0,0.35,0)$ in the case of the incident evanescent wave.

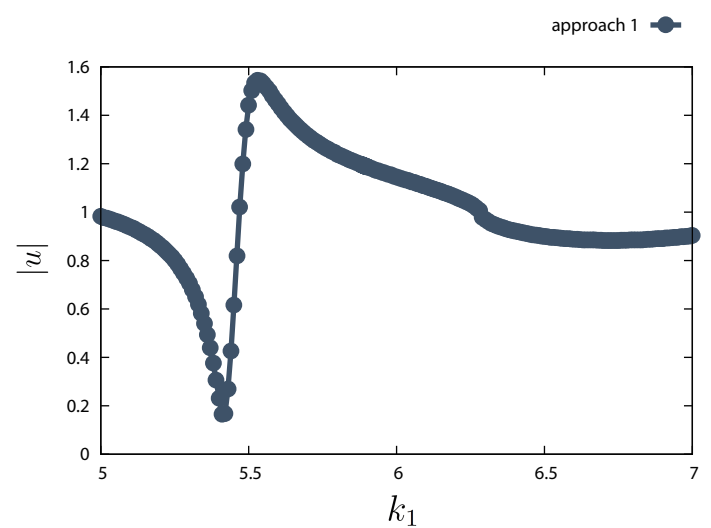

Figure 10: The value of $|u|$ at $\boldsymbol{x} \approx(-0.35,0,0)$ in the case of the incident plane wave. 

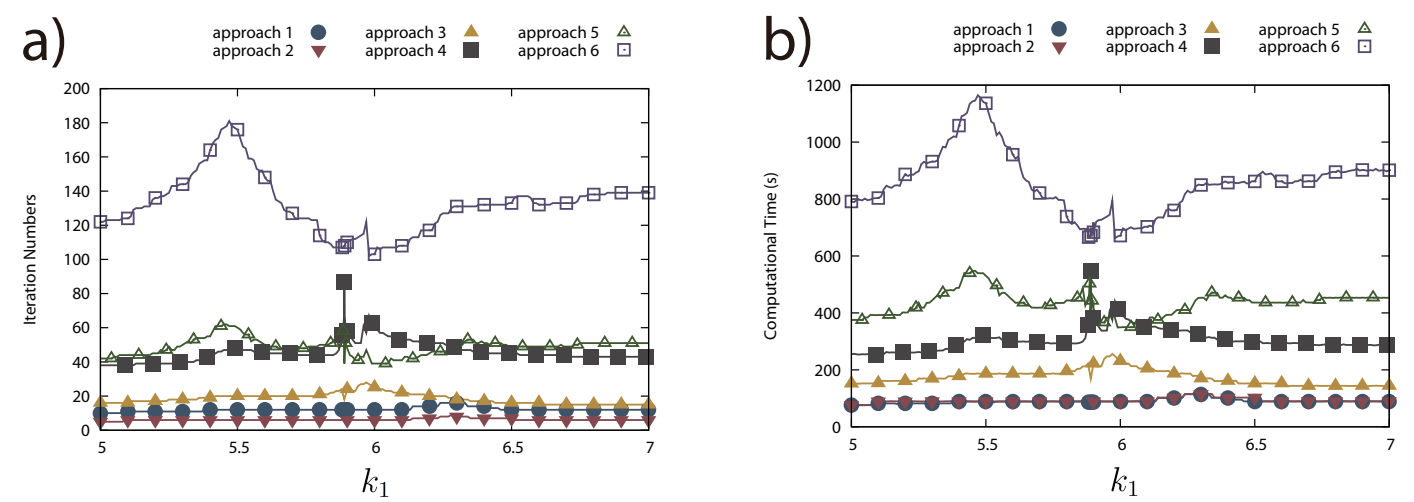

Figure 11: The number of iterations and the computational time of GMRES and FGMRES for the model of spheres around embedded guided modes. a) The number of iterations b) The computational time

uniqueness of the solution does not hold at singular frequencies. Fig. 12 also shows that this non-uniqueness occurs only right at this frequency, and the solution remains on the solution curve elsewhere around $k_{1}=5.89$ and 5.97.

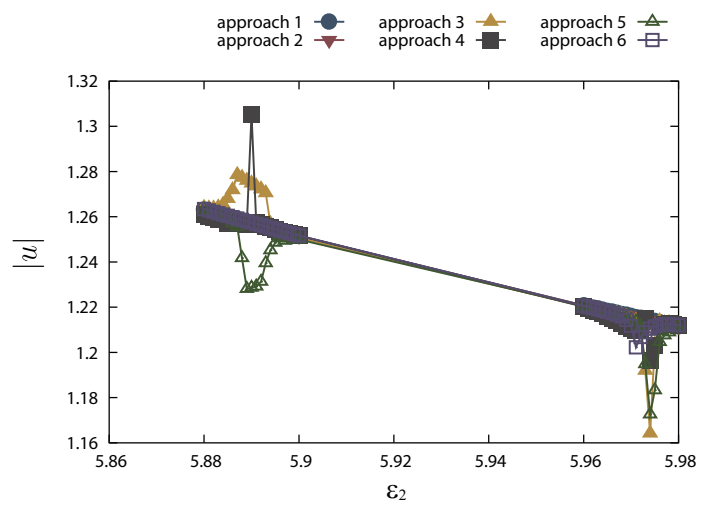

Figure 12: The value of $|u|$ at $\boldsymbol{x} \approx(-0.35,0,0)$ in the case of the incident plane wave.

The performances of the Calderon preconditioners for still higher frequencies and finer mesh are investigated in Table 2, where the numbers of matrix-vector products required by the 6 approaches are given. The parameters are the same as in the previous example except for the frequency. The 
incident wave is planar. This table shows more or less the same tendencies as have been observed in Fig. 11. We also see that the Calderon preconditioners (approaches 1 and 2) remain effective with finer meshes.

Table 2: Number of matrix-vector products for the model of spheres.

\begin{tabular}{|c|c|c|c|c|c|c|c|}
\hline degrees of freedom & \multicolumn{5}{|c|}{5784} & 5784 & 172984 \\
\hline$k_{1}$ & 10 & 15 & 20 & 25 & 30 & \multicolumn{2}{|c|}{5.5} \\
\hline approach 1 & 3 & 3 & 3 & 3 & 5 & 12 & 12 \\
\hline approach 2 & 4 & 4 & 4 & 4 & 6 & 12 & 12 \\
\hline approach 3 & 18 & 22 & 31 & 35 & 21 & 20 & 87 \\
\hline approach 4 & 31 & 29 & 29 & 27 & 27 & 48 & 51 \\
\hline approach 5 & 10 & 80 & 123 & 188 & 194 & 60 & 86 \\
\hline approach 6 & 161 & 227 & 303 & 451 & 419 & 176 & 402 \\
\hline
\end{tabular}

Summing up, we conclude that preconditioning approaches based on Calderon's formulae (approaches 1 and 2) are effective in reducing the number of iterations even near guided modes and anomalies.

\subsection{Computational time for more complicated domains}

To examine the effectiveness of the Calderon preconditioners in problems with complicated domains, we solved the problem for the fish model shown in Fig. 13 a) subject to a plane incident wave. The constants are set as follows: $\varepsilon_{1}=1, \varepsilon_{2}=2.56, \omega=28, \beta_{2}=1$ and $\beta_{3}=0$. The length of the model from mouth to tail fin is about 0.6. The degree of freedom is 34308. The computed values of $|u|$ on the boundary are shown in Fig. 13 b). Table 3 gives the iteration numbers and the computational time for the 6 approaches in this problem. Items " $>1000$ " in this table mean that the residual did not decrease less than the given error tolerance $10^{-4}$ after 1000 matrix-vector product operations. Once again, we see that the Calderon preconditioners are effective in reducing iteration numbers and computational time.

\section{Conclusion}

We have verified that the preconditioning approaches based on Calderon's formulae are effective in the periodic FMM for transmission boundary value problems for Helmholtz equation discretised with the Galerkin method. Although we cannot precondition Galerkin BEM matrices just by ordering the 

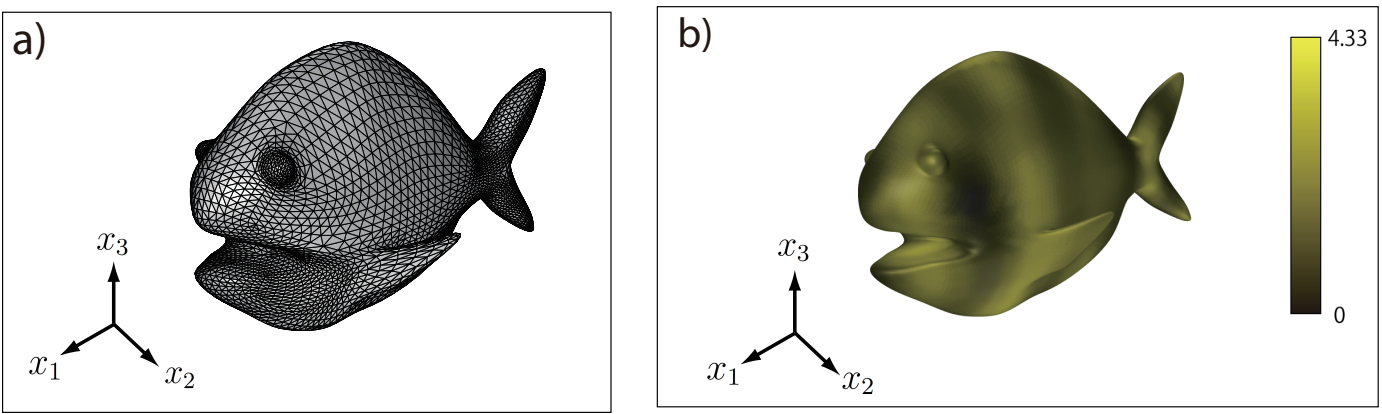

Figure 13: a) The model of fish (unit cell). b) The value of $|u|$ on the boundary in the model of fish.

Table 3: Number of matrix-vector products for the model of fish

\begin{tabular}{|c|c|c|}
\hline & matrix-vector products & computational time(s) \\
\hline approach 1 & 58 & 2422 \\
\hline approach 2 & 58 & 2420 \\
\hline approach 3 & 462 & 29900 \\
\hline approach 4 & $>1000$ & 33029 \\
\hline approach 5 & 505 & \\
\hline approach 6 & $>1000$ & \\
\hline
\end{tabular}


unknowns and equations appropriately as we could with collocation, we can still find a fairly simple preconditioners based on Calderon's formulae in the Galerkin cases. Specifically, we can use the Grammian matrix as a preconditioner (approach 1) if we use the right ordering of equations and unknowns. The inverse of this preconditioner is obtained very easily with iterative methods since this matrix is well-conditioned. We also have another implementation of the Calderon preconditioning (approach 2), which is slightly less efficient in terms of the computational time but requires less memory than the approach 1. Furthermore, it is found that the preconditioners based on Calderon's formulae can decrease the iteration number even near frequencies where the solution shows anomalous behaviours related to the periodicity of the problems.

In this paper, however, we tested the efficiency of the Calderon preconditioners only in simple academic problems. Applying this method to more complicated problems found in real world applications is an important future plan. Applications of the proposed preconditioning approaches to other equations such as Maxwell's equations and the elastic wave equation also remain to be investigated. It is known that a naive application of the preconditioning based on Calderon's formulae for Maxwell's equations in 3D makes the coefficient matrix singular[18]. In addition, the square of the integral operator which appears in the PMCHWT formulation for Maxwell's equations does not take the form of the identity plus a compact operator[13]. The same conclusion holds in elasticity as well. However, this does not necessarily mean that approaches similar to those presented in this paper are ineffective in Maxwell's equations or in elasticity. The reader is referred to [19] for further details in elasticity. Our approach in Maxwell's equations will be presented in future publications.

\section{References}

[1] J. Joannopoulos, R. Meade, J. Winn, Photonic Crystals, Princeton University Press, Princeton, NJ, USA, 1995.

[2] J. Pendry, Negative refraction makes a perfect lens, Physical Review Letters 85 (2000) 3966-3969.

[3] Y. Otani, N. Nishimura, A periodic FMM for Maxwell's equations in 3D and its applications to problems related to photonic crystals, Journal of Computational Physics 227 (9) (2008) 4630-4652. 
[4] A. Barnett, L. Greengard, A new integral representation for quasiperiodic fields and its application to two-dimensional band structure calculations, Journal of Computational Physics 229 (19) (2010) 6898-6914.

[5] R. Petit, L. Botten, Electromagnetic Theory of Gratings, SpringerVerlag Berlin, 1980.

[6] Y. Otani, N. Nishimura, Behaviour of periodic fast multipole boundary integral equation method for Maxwell's equations near Wood's anomalies, in: H. Ammari, H.-B. Kang (Eds.), Contemporary Mathematics 494, AMS, 2009, pp. 43-59.

[7] Y. Saad, Iterative Methods for Sparse Linear Systems, Society for Industrial and Applied Mathematics, Philadelphia, PA, USA, 2003.

[8] O. Steinbach, W. Wendland, The construction of some efficient preconditioners in the boundary element method, Advances in Computational Mathematics 9 (1) (1998) 191-216.

[9] S. Christiansen, J. Nédélec, Des préconditionneurs pour la résolution numérique des équations intégrales de frontière de l'acoustique, Comptes Rendus de l'Academie des Sciences-Série I-Mathématique 330 (7) (2000) 617-622.

[10] X. Antoine, Y. Boubendir, An integral preconditioner for solving the two-dimensional scattering transmission problem using integral equations, International Journal of Computer Mathematics 85 (10) (2008) $1473-1490$.

[11] A. Buffa, S. Christiansen, A dual finite element complex on the barycentric refinement, Mathematics of Computation 76 (2007) 1743-1769.

[12] F. Andriulli, K. Cools, H. Bagci, F. Olyslager, A. Buffa, S. Christiansen, E. Michielssen, A multiplicative Calderón preconditioner for the electric field integral equation, IEEE Transactions on Antennas and Propagation 56 (8) (2008) 2398-2412.

[13] S. Yan, J. Jin, Z. Nie, A comparative study of Calderón preconditioners for PMCHWT equations, IEEE Transactions Antennas Propagation 58 (7) (2010) 2375-2383. 
[14] K. Niino, N. Nishimura, Preconditioning based on Calderon's formulae for 1 periodic boundary value problems for Helmholtz' equation in 2D (in Japanese), Transactions of the Japan Society for Computational Methods in Engineering 9 (2009) 1-6.

[15] Y. Otani, N. Nishimura, An FMM for periodic boundary value problems for cracks for Helmholtz equation in 2D, International Journal for Numerical Methods in Engineering 73 (3) (2008) 381.

[16] A.-S. Bonnet-Bendhia, F. Starling, Guided waves by electromagnetic gratings and non-uniqueness examples for the diffraction problems, Mathematical Methods in the Applied Sciences 17 (1994) 305-338.

[17] S. Shipman, D. Volkov, Existence of guided modes on periodic slabs, Discrete and Continuous Dynamical Systems, Supplement Volume (2005) 784-791.

[18] S. Christiansen, J. Nédélec, A preconditioner for the electric field integral equation based on Calderon formulas, SIAM Journal on Numerical Analysis 40 (3) (2003) 1100-1135.

[19] H. Isakari, K. Niino, H. Yoshikawa, N. Nishimura, Calderon's preconditioning for periodic FMM for elastodynamics in 3D, accepted for publication in International Journal for Numerical Methods in Engineering (2011). 